\title{
Iterative LMMSE Transceiver Design for Dual-Hop AF MIMO Relay Systems Under Channel Uncertainties
}

\author{
Chengwen Xing, Shaodan Ma and Yik-Chung Wu \\ Department of Electrical and Electronic Engineering \\ The University of Hong Kong, Hong Kong \\ Email: \{cwxing, sdma, ycwu\}@eee.hku.hk
}

\begin{abstract}
This paper considers the problem of robust linear transceiver design for a dual-hop amplify-and-forward (AF) MIMO relay system, with Gaussian random channel uncertainties in both hops. By taking the channel uncertainties into account, an iterative algorithm is proposed to minimize the mean-square-error (MSE) of the output signal at the destination. Simulation results show that the proposed algorithm reduces the sensitivity of the AF MIMO relay systems to channel estimation errors, and performs better than the algorithm based on estimated channels only.
\end{abstract}

\section{INTRODUCTION}

Due to its great potentials to improve reliability, coverage and capacity of wireless links [1], cooperative communications has gained significant research interest. Generally speaking, there are three kinds of cooperative protocols: amplify-andforward (AF), compress-and-forward (CF) and decode-andforward (DF). Among these three protocols, AF is a relatively simple one, in which the relay just scales the signal received from the source, and then transmits it to the destination. Because of its simplicity and low implementation complexity, AF strategy has attracted a lot of researchers' attention.

On the other hand, multiantenna systems have been extensively studied in the past decades, and it is well-known that in fully scattered environment, multiantenna systems can provide substantial spatial diversity and multiplexing gains [2]. In particular, the benefits of multiantenna systems can be directly introduced into cooperative communications via deployment of multiple antennas at transmitters and receivers.

For dual-hop AF MIMO relay systems with single relay, the optimal closed-form solution for joint LMMSE transceiver design assuming perfect channel state information (CSI) has been proposed in [4]. It has also been shown in [4] that the joint design has a better performance than the various separate design schemes. In [5], joint LMMSE transceiver design for multiple-relay case has been discussed. After a relaxation on the constraint of the transmit power at the relay, a suboptimal closed-form solution has been derived in [5].

Notice that the above mentioned algorithms on LMMSE transceiver design for AF MIMO relay systems require the CSI being perfectly known. Unfortunately, in practice, CSI is generally obtained through estimation and perfect estimation

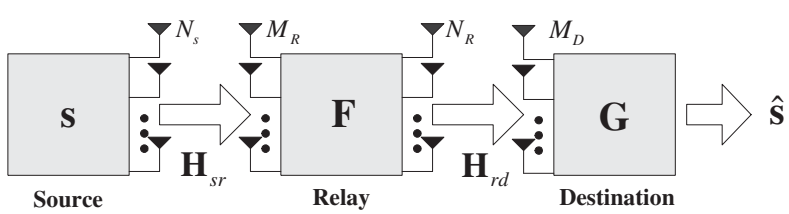

Fig. 1. Amplify-and-forward MIMO relay.

is very difficult to achieve. Robust transceiver design, which could mitigate such performance degradation by taking the channel estimation errors into account, is therefore of great importance and highly desirable for practical applications.

In this paper, we consider an AF MIMO relay system with single relay and address the problem of robust transceiver design under imperfect CSI at both the relay and destination. With the channel estimation errors being modeled as Gaussian random variables, robustness is incorporated into the optimization objective function in the form of MSE averaged over the channel estimation errors. Simulation results show that the proposed robust algorithm performs better than the transceiver without taking channel estimation errors into account.

The following notations are used throughout this paper. Boldface lowercase letters denote vectors, while boldface uppercase letters denote matrices. The notations $\mathbf{Z}^{\mathrm{T}}, \mathbf{Z}^{\mathrm{H}}$ and $\mathbf{Z}^{*}$ denote the transpose, Hermitian and conjugate of the matrix $\mathbf{Z}$, respectively, and $\operatorname{Tr}(\mathbf{Z})$ is the trace of the matrix $\mathbf{Z}$. The symbol $\mathbf{I}_{M}$ denotes the $M \times M$ identity matrix, while $\mathbf{0}_{M \times N}$ denotes the $M \times N$ all zero matrix. The symbol $\mathbb{E}\{$.$\} represents$ the expectation operation. The operation $\operatorname{det}(\mathbf{Z})$ denotes the determinant of $\mathbf{Z}$.

\section{SyStem MODEL}

In this paper, a dual-hop amplify-and-forward (AF) cooperative communication system is considered. In the considered system, there is one source with $N_{S}$ antennas, one relay with $M_{R}$ receive antennas and $N_{R}$ transmit antennas, and one destination with $M_{D}$ antennas, as shown in Fig. 1. At the first hop, the source transmits data to the relay. The received signal, $\mathbf{x}$, at the relay is

$$
\mathbf{x}=\mathbf{H}_{s r} \mathbf{s}+\mathbf{n}_{1}
$$


where $\mathbf{s}$ is the $N_{S} \times 1$ data vector transmitted by the source with the covariance matrix $\mathbf{R}_{s}=\mathbb{E}\left\{\mathbf{s s}^{\mathrm{H}}\right\}$. The matrix $\mathbf{H}_{s r}$ is the MIMO channel matrix between the source and the relay. Symbol $\mathbf{n}_{1}$ is the additive Gaussian noise with covariance matrix $\mathbf{R}_{n_{1}}$. At the relay, the received signal $\mathbf{x}$ is multiplied by a precoder matrix $\mathbf{F}$, under a power constraint $\operatorname{Tr}\left(\mathbf{F R}_{\mathbf{x}} \mathbf{F}^{\mathrm{H}}\right) \leq$ $P_{r}$ where $\mathbf{R}_{\mathbf{x}}=\mathbb{E}\left\{\mathbf{x x}^{\mathrm{H}}\right\}$ and $P_{r}$ is the maximum transmit power. Then the resulting signal is transmitted to the destination. The received signal at the destination, $\mathbf{y}$, can be written as

$$
\mathbf{y}=\mathbf{H}_{r d} \mathbf{F} \mathbf{H}_{s r} \mathbf{s}+\mathbf{H}_{r d} \mathbf{F} \mathbf{n}_{1}+\mathbf{n}_{2},
$$

where $\mathbf{H}_{r d}$ is the MIMO channel matrix between the relay and the destination, and $\mathbf{n}_{2}$ is the additive Gaussian noise vector at the second hop with covariance matrix $\mathbf{R}_{n_{2}}$. In order to guarantee the transmitted data $\mathbf{s}$ can be recovered at the destination, it is assumed that $M_{R}, N_{R}$, and $M_{D}$ are greater than or equal to $N_{S}$ [5].

It is assumed that both the relay and destination have the estimated channel state information (CSI). When channel estimation errors are considered, we have

$$
\begin{aligned}
\mathbf{H}_{s r} & =\overline{\mathbf{H}}_{s r}+\Delta \mathbf{H}_{s r}, \\
\mathbf{H}_{r d} & =\overline{\mathbf{H}}_{r d}+\Delta \mathbf{H}_{r d},
\end{aligned}
$$

where $\overline{\mathbf{H}}_{s r}$ and $\overline{\mathbf{H}}_{r d}$ are the estimated CSI, while $\Delta \mathbf{H}_{s r}$ and $\Delta \mathbf{H}_{r d}$ are the corresponding channel estimation errors whose elements are zero mean Gaussian random variables. In general, the $M_{R} \times N_{S}$ matrix $\Delta \mathbf{H}_{s r}$ can be written as $\Delta \mathbf{H}_{s r}=\boldsymbol{\Sigma}_{s r}^{\frac{1}{2}} \mathbf{H}_{W, s r} \boldsymbol{\Psi}_{s r}^{\frac{1}{2}}$, where the elements of the $M_{R} \times$ $N_{S}$ matrix $\mathbf{H}_{W, s r}$ are independent and identically distributed (i.i.d.) Gaussian random variables with zero mean and unit variance. The $M_{R} \times M_{R}$ matrix $\boldsymbol{\Sigma}_{s r}$ and $N_{S} \times N_{S}$ matrix $\Psi_{s r}^{\mathrm{T}}$ are the row and column covariance matrices of $\Delta \mathbf{H}_{s r}$, respectively [2]. The matrix $\Delta \mathbf{H}_{s r}$ is said to have a matrixvariate complex Gaussian distribution, which can be written as [6]

$$
\Delta \mathbf{H}_{s r} \sim \mathcal{C N}_{M_{R}, N_{S}}\left(\mathbf{0}_{M_{R} \times N_{S}}, \boldsymbol{\Sigma}_{s r} \otimes \mathbf{\Psi}_{s r}^{\mathrm{T}}\right),
$$

with the probability density function (p.d.f.) given by [7]

$$
p\left(\Delta \mathbf{H}_{s r}\right)=\frac{\exp \left(-\operatorname{Tr}\left(\Delta \mathbf{H}_{s r}^{\mathrm{H}} \boldsymbol{\Sigma}_{s r}^{-1} \Delta \mathbf{H}_{s r} \boldsymbol{\Psi}_{s r}^{-1}\right)\right)}{(\pi)^{N_{S} M_{R}}\left[\operatorname{det}\left(\boldsymbol{\Sigma}_{s r}\right)\right]^{N_{S}}\left[\operatorname{det}\left(\boldsymbol{\Psi}_{s r}\right)\right]^{M_{R}}} .
$$

Similarly, for the estimation error in the second hop, we have

$$
\Delta \mathbf{H}_{r d} \sim \mathcal{C N}_{M_{D}, N_{R}}\left(\mathbf{0}_{M_{D} \times N_{R}}, \boldsymbol{\Sigma}_{r d} \otimes \mathbf{\Psi}_{r d}^{\mathrm{T}}\right)
$$

where the $M_{D} \times M_{D}$ matrix $\boldsymbol{\Sigma}_{r d}$ and $N_{R} \times N_{R}$ matrix $\boldsymbol{\Psi}_{r d}^{\mathrm{T}}$ are the row and column covariance matrices of $\Delta \mathbf{H}_{r d}$, respectively. It is assumed that $\mathbf{H}_{s r}$ and $\mathbf{H}_{r d}$ are estimated independently, so the channel estimation errors, $\Delta \mathbf{H}_{s r}$ and $\Delta \mathbf{H}_{r d}$, are independent.

\section{PROBlem Formulation}

At the destination, a linear equalizer $\mathbf{G}$ is adopted to detect the transmitted data $\mathbf{S}$ (see Fig. 1). The problem is how to design the linear precoder matrix $\mathbf{F}$ at the relay and the linear equalizer $\mathbf{G}$ at the destination to minimize the mean-squareerror (MSE) of the received data at the destination:

$$
\operatorname{MSE}(\mathbf{F}, \mathbf{G})=\mathbb{E}\left\{\operatorname{Tr}\left((\mathbf{G y}-\mathbf{s})(\mathbf{G y}-\mathbf{s})^{\mathrm{H}}\right)\right\},
$$

where the expectation is taken with respect to s, $\Delta \mathbf{H}_{s r}, \Delta \mathbf{H}_{r d}$, $\mathbf{n}_{1}$ and $\mathbf{n}_{2}$.

Since $\mathbf{s}, \mathbf{n}_{1}$ and $\mathbf{n}_{2}$ are independent, the MSE expression (7) can be written as

$$
\begin{aligned}
& \operatorname{MSE}(\mathbf{F}, \mathbf{G}) \\
= & \mathbb{E}\left\{\left\|\left(\mathbf{G H}_{r d} \mathbf{F} \mathbf{H}_{s r}-\mathbf{I}_{N_{S}}\right) \mathbf{s}+\mathbf{G H}_{r d} \mathbf{F} \mathbf{n}_{1}+\mathbf{G n}_{2}\right\|^{2}\right\} \\
= & \mathbb{E}_{\Delta \mathbf{H}_{s r}, \Delta \mathbf{H}_{r d}}\left\{\operatorname { T r } \left(\left(\mathbf{G H}_{r d} \mathbf{F} \mathbf{H}_{s r}-\mathbf{I}_{N_{S}}\right) \mathbf{R}_{s}\right.\right. \\
& \left.\quad \times\left(\mathbf{G H}_{r d} \mathbf{F} \mathbf{H}_{s r}-\mathbf{I}_{N_{S}}\right)^{\mathrm{H}}\right\} \\
& +\mathbb{E}_{\Delta \mathbf{H}_{r d}}\left\{\operatorname{Tr}\left(\left(\mathbf{G} \mathbf{H}_{r d} \mathbf{F}\right) \mathbf{R}_{n_{1}}\left(\mathbf{G} \mathbf{H}_{r d} \mathbf{F}\right)^{\mathrm{H}}\right)\right\} \\
& +\operatorname{Tr}\left(\mathbf{G R}_{n_{2}} \mathbf{G}^{\mathrm{H}}\right) \\
= & \mathbb{E}_{\Delta \mathbf{H}_{s r}, \Delta \mathbf{H}_{r d}}\left\{\operatorname{Tr}\left(\left(\mathbf{G H}_{r d} \mathbf{F} \mathbf{H}_{s r}\right) \mathbf{R}_{s}\left(\mathbf{G H}_{r d} \mathbf{F} \mathbf{H}_{s r}\right)^{\mathrm{H}}\right)\right\} \\
& +\operatorname{Tr}\left(\mathbf{G E}_{\Delta \mathbf{H}_{r d}}\left\{\mathbf{H}_{r d} \mathbf{F} \mathbf{R}_{n_{1}} \mathbf{F}^{\mathrm{H}} \mathbf{H}_{r d}^{\mathrm{H}}\right\} \mathbf{G}^{\mathrm{H}}\right) \\
& -\operatorname{Tr}\left(\mathbf{R}_{s}\left(\mathbf{G} \overline{\mathbf{H}}_{r d} \mathbf{F} \overline{\mathbf{H}}_{s r}\right)^{\mathrm{H}}\right)-\operatorname{Tr}\left(\mathbf{G} \overline{\mathbf{H}}_{r d} \mathbf{F} \overline{\mathbf{H}}_{s r} \mathbf{R}_{s}\right) \\
& +\operatorname{Tr}\left(\mathbf{R}_{s}\right)+\operatorname{Tr}\left(\mathbf{G R}_{n_{2}} \mathbf{G}^{\mathrm{H}}\right) .
\end{aligned}
$$

Because $\Delta \mathbf{H}_{s r}$ and $\Delta \mathbf{H}_{r d}$ are independent, the first term of MSE is

$$
\begin{aligned}
& \mathbb{E}_{\Delta \mathbf{H}_{s r}, \Delta \mathbf{H}_{r d}}\left\{\operatorname{Tr}\left(\left(\mathbf{G H}_{r d} \mathbf{F} \mathbf{H}_{s r}\right) \mathbf{R}_{s}\left(\mathbf{G H}_{r d} \mathbf{F} \mathbf{H}_{s r}\right)^{\mathrm{H}}\right)\right\} \\
& =\operatorname{Tr}\left(\mathbf{G E}_{\Delta \mathbf{H}_{r d}}\left\{\mathbf{H}_{r d} \mathbf{F} \mathbb{E}_{\Delta \mathbf{H}_{s r}}\left\{\mathbf{H}_{s r} \mathbf{R}_{s} \mathbf{H}_{s r}^{\mathrm{H}}\right\} \mathbf{F}^{\mathrm{H}} \mathbf{H}_{r d}^{\mathrm{H}}\right\} \mathbf{G}^{\mathrm{H}}\right) \text {. }
\end{aligned}
$$

For the inner expectation, due to the fact that the distribution of $\Delta \mathbf{H}_{s r}$ is matrix-variate complex Gaussian with zero mean, the following equation holds [6]

$$
\begin{aligned}
& \mathbb{E}_{\Delta \mathbf{H}_{s r}}\left\{\mathbf{H}_{s r} \mathbf{R}_{s} \mathbf{H}_{s r}^{\mathrm{H}}\right\} \\
& =\mathbb{E}_{\Delta \mathbf{H}_{s r}}\left\{\left(\overline{\mathbf{H}}_{s r}+\Delta \mathbf{H}_{s r}\right) \mathbf{R}_{s}\left(\overline{\mathbf{H}}_{s r}+\Delta \mathbf{H}_{s r}\right)^{\mathrm{H}}\right\} \\
& =\operatorname{Tr}\left(\mathbf{R}_{s} \boldsymbol{\Psi}_{s r}\right) \boldsymbol{\Sigma}_{s r}+\overline{\mathbf{H}}_{s r} \mathbf{R}_{\mathrm{s}} \overline{\mathbf{H}}_{s r}^{\mathrm{H}} \\
& \triangleq \boldsymbol{\Pi}_{0} .
\end{aligned}
$$

Applying (10) and the corresponding result for $\Delta \mathbf{H}_{r d}$ to (9), the first term of MSE becomes

$$
\begin{aligned}
& \operatorname{Tr}\left(\mathbf{G} \mathbb{E}_{\Delta \mathbf{H}_{r d}}\left\{\mathbf{H}_{r d} \mathbf{F} \mathbb{E}_{\Delta \mathbf{H}_{s}}\left\{\mathbf{H}_{s r} \mathbf{R}_{s} \mathbf{H}_{s r}^{\mathrm{H}}\right\} \mathbf{F}^{\mathrm{H}} \mathbf{H}_{r d}^{\mathrm{H}}\right\} \mathbf{G}^{\mathrm{H}}\right) \\
& =\operatorname{Tr}\left(\mathbf{G}\left(\operatorname{Tr}\left(\mathbf{F} \boldsymbol{\Pi}_{0} \mathbf{F}^{\mathrm{H}} \mathbf{\Psi}_{r d}\right) \boldsymbol{\Sigma}_{r d}+\overline{\mathbf{H}}_{r d} \mathbf{F} \boldsymbol{\Pi}_{0} \mathbf{F}^{\mathrm{H}} \overline{\mathbf{H}}_{r d}^{\mathrm{H}}\right) \mathbf{G}^{\mathrm{H}}\right) .
\end{aligned}
$$

Similarly, the second term of MSE in (8) can be simplified as

$$
\begin{aligned}
& \operatorname{Tr}\left(\mathbf{G E}_{\Delta \mathbf{H}_{r d}}\left\{\mathbf{H}_{r d} \mathbf{F} \mathbf{R}_{n_{1}} \mathbf{F}^{\mathrm{H}} \mathbf{H}_{r d}^{\mathrm{H}}\right\} \mathbf{G}^{\mathrm{H}}\right) \\
& =\operatorname{Tr}\left(\mathbf{G}\left(\operatorname{Tr}\left(\mathbf{F} \mathbf{R}_{\mathrm{n}_{1}} \mathbf{F}^{\mathrm{H}} \mathbf{\Psi}_{r d}\right) \boldsymbol{\Sigma}_{r d}+\overline{\mathbf{H}}_{r d} \mathbf{F} \mathbf{R}_{n_{1}} \mathbf{F}^{\mathrm{H}} \overline{\mathbf{H}}_{r d}^{\mathrm{H}}\right) \mathbf{G}^{\mathrm{H}}\right) .
\end{aligned}
$$

Based on (11) and (12), the MSE (8) equals to

$$
\begin{aligned}
\operatorname{MSE}(\mathbf{F}, \mathbf{G})= & \operatorname{Tr}\left(\mathbf{G}\left(\overline{\mathbf{H}}_{r d} \mathbf{F} \mathbf{R}_{\mathbf{x}} \mathbf{F}^{\mathrm{H}} \overline{\mathbf{H}}_{r d}^{\mathrm{H}}+\mathbf{K}\right) \mathbf{G}^{\mathrm{H}}\right) \\
& -\operatorname{Tr}\left(\mathbf{R}_{s} \overline{\mathbf{H}}_{s r}^{\mathrm{H}} \mathbf{F}^{\mathrm{H}} \overline{\mathbf{H}}_{r d}^{\mathrm{H}} \mathbf{G}^{\mathrm{H}}\right) \\
& -\operatorname{Tr}\left(\mathbf{R}_{s} \mathbf{G} \overline{\mathbf{H}}_{r d} \mathbf{F} \overline{\mathbf{H}}_{s r}\right)+\operatorname{Tr}\left(\mathbf{R}_{s}\right)
\end{aligned}
$$


where

$$
\begin{aligned}
\mathbf{R}_{\mathbf{x}} & =\mathbb{E}\left\{\mathbf{x x}^{\mathrm{H}}\right\}=\boldsymbol{\Pi}_{0}+\mathbf{R}_{n_{1}} \\
\mathbf{K} & =\operatorname{Tr}\left(\mathbf{F}\left(\boldsymbol{\Pi}_{0}+\mathbf{R}_{n_{1}}\right) \mathbf{F}^{\mathrm{H}} \boldsymbol{\Psi}_{r d}\right) \boldsymbol{\Sigma}_{r d}+\mathbf{R}_{n_{2}} .
\end{aligned}
$$

Notice that the matrix $\mathbf{R}_{\mathrm{x}}$ is the autocorrelation matrix of the receive signal $\mathrm{x}$ at the relay.

Subject to the transmit power constraint at the relay, the joint design of equalizer at the destination and precoder at the relay can be expressed as the following optimization problem

$$
\begin{array}{cl}
\min _{\mathbf{F}, \mathbf{G}} & \operatorname{MSE}(\mathbf{F}, \mathbf{G}) \\
\text { s.t. } & \operatorname{Tr}\left(\mathbf{F R}_{\mathbf{x}} \mathbf{F}^{\mathrm{H}}\right) \leq P_{r} .
\end{array}
$$

If the channels are perfectly known without estimation errors, the problem (16) has been solved in [4]. However, perfect channel estimation is difficult to achieve in practice, due to limited training and time-varying nature of wireless channels. Channel estimation errors generally exist and the formulation (13) is a very complicated function of $\mathbf{F}$ and $\mathbf{G}$, making the problem difficult to solve.

\section{The Proposed Iterative Algorithm}

In this section, we derive an iterative algorithm to solve for $\mathbf{F}$ and $\mathbf{G}$. In the following, it is shown that given $\mathbf{F}$, there is a closed-form solution for $\mathbf{G}$, and vice versa. Therefore, the proposed algorithm computes $\mathbf{F}$ and $\mathbf{G}$ iteratively, starting with an initial value.

\section{A. Updating $\mathbf{G}$ given $\mathbf{F}$}

Suppose the solutions for $\mathbf{F}$ and $\mathbf{G}$ at the $i^{\text {th }}$ iteration are $\mathbf{F}_{i}$ and $\mathbf{G}_{i}$, respectively. First we update $\mathbf{G}$ for given $\mathbf{F}=\mathbf{F}_{i}$. As the constraint in (16) is independent of $\mathbf{G}$, at the $(i+1)^{\text {th }}$ iteration, the optimal $\mathbf{G}_{i+1}$ for given $\mathbf{F}_{i}$ satisfies the following condition

$$
\frac{\partial \operatorname{MSE}\left(\mathbf{F}_{i}, \mathbf{G}_{i+1}\right)}{\partial \mathbf{G}_{i+1}^{*}}=0,
$$

based on which we have

$$
\mathbf{G}_{i+1}=\mathbf{R}_{s}\left(\overline{\mathbf{H}}_{r d} \mathbf{F}_{i} \overline{\mathbf{H}}_{s r}\right)^{\mathrm{H}}\left(\overline{\mathbf{H}}_{r d} \mathbf{F}_{i} \mathbf{R}_{\mathbf{x}} \mathbf{F}_{i}^{\mathrm{H}} \overline{\mathbf{H}}_{r d}^{\mathrm{H}}+\mathbf{K}_{i}\right)^{-1},
$$

where $\mathbf{K}_{i}=\operatorname{Tr}\left(\mathbf{F}_{i}\left(\boldsymbol{\Pi}_{0}+\mathbf{R}_{n_{1}}\right) \mathbf{F}_{i}^{\mathrm{H}} \boldsymbol{\Psi}_{r d}\right) \boldsymbol{\Sigma}_{r d}+\mathbf{R}_{n_{2}}$.

\section{B. Updating $\mathbf{F}$ given $\mathbf{G}$}

When $\mathbf{G}_{i+1}$ is obtained as (18), we can design $\mathbf{F}_{i+1}$ based on KKT conditions, which are obtained by differentiating the Lagrangian function of (16), $\mathcal{L}\left(\mathbf{F}_{i+1}, \gamma_{i+1}\right)=$ $\operatorname{MSE}\left(\mathbf{F}_{i+1}, \mathbf{G}_{i+1}\right)+\gamma_{i+1}\left(\operatorname{Tr}\left(\mathbf{F}_{i+1} \mathbf{R}_{\mathbf{x}} \mathbf{F}_{i+1}^{\mathrm{H}}\right)-P_{r}\right)$, with respect to $\mathbf{F}_{i+1}^{*}$. The KKT conditions can be shown to be

$$
\begin{aligned}
& \mathbf{F}_{i+1}=\left(\overline{\mathbf{H}}_{r d}^{\mathrm{H}} \mathbf{G}_{i+1}^{\mathrm{H}} \mathbf{G}_{i+1} \overline{\mathbf{H}}_{r d}+\operatorname{Tr}\left(\mathbf{G}_{i+1} \boldsymbol{\Sigma}_{r d} \mathbf{G}_{i+1}^{\mathrm{H}}\right) \mathbf{\Psi}_{r d}\right. \\
& \left.+\gamma_{i+1} \mathbf{I}\right)^{-1} \overline{\mathbf{H}}_{r d}^{\mathrm{H}} \mathbf{G}_{i+1}^{\mathrm{H}} \mathbf{R}_{s} \overline{\mathbf{H}}_{s r}^{\mathrm{H}} \mathbf{R}_{\mathbf{x}}^{-1}, \\
& \gamma_{i+1}\left[\operatorname{Tr}\left(\mathbf{F}_{i+1} \mathbf{R}_{\mathbf{x}} \mathbf{F}_{i+1}^{\mathrm{H}}\right)-P_{r}\right]=0, \quad \gamma_{i+1} \geq 0, \\
& \operatorname{Tr}\left(\mathbf{F}_{i+1} \mathbf{R}_{\mathbf{x}} \mathbf{F}_{i+1}^{\mathrm{H}}\right)-P_{r} \leq 0,
\end{aligned}
$$

where $\gamma_{i+1}$ is the Lagrangian multiplier corresponding to $\mathbf{F}_{i+1}$ at the $(i+1)^{\text {th }}$ iteration. Notice that when $\mathbf{G}_{i+1}$ is fixed, the optimization problem (16) is a convex quadratic programming
Algorithm 1 The iterative algorithm for joint design of $\mathbf{F}$ and G

Initialize the algorithm with $\mathbf{F}_{0}$ where the $N \times N$ principle sub-matrix of $\mathbf{F}_{0}$ being $\sqrt{\frac{P_{r}}{\operatorname{Tr}\left(\mathbf{R}_{\mathbf{x}}\right)}} \mathbf{I}_{N}$, and $N=$ $\min \left\{N_{R}, M_{R}\right\}$, while the remaining entries of $\mathbf{F}_{0}$ being zeros.

repeat

Update $\mathbf{G}_{i}$ using (18);

Compute $\gamma_{i}$ using (22);

Update $\mathbf{F}_{i}$ using (19a);

$i=i+1$;

until $\left\|\mathbf{F}_{i}-\mathbf{F}_{i-1}\right\|^{2}$ is smaller than a threshold $\epsilon$, where the index $i$ denotes the $i^{\text {th }}$ iteration.

problem for $\mathbf{F}_{i+1}$, the KKT conditions are the necessary and sufficient conditions for the optimal $\mathbf{F}_{i+1}$ [9].

Obviously from (19a), in order to compute the optimal $\mathbf{F}_{i+1}$, the Lagrangian multiplier $\gamma_{i+1}$ should be calculated first. However, there is no closed-form solution of $\gamma_{i+1}$ simultaneously satisfying (19b) and (19c) [10]. Below we propose a low complexity method to solve (19b) and (19c). First, notice that in order to have (19b) satisfied, either $\gamma_{i+1}=0$ or $\operatorname{Tr}\left(\mathbf{F}_{i+1} \mathbf{R}_{\mathbf{x}} \mathbf{F}_{i+1}^{\mathrm{H}}\right)=P_{r}$ must hold. If $\gamma_{i+1}=0$ also makes (19c) satisfied, $\gamma_{i+1}=0$ is a solution to (19b) and (19c). Since given $\mathbf{G}_{i+1}$, the optimization problem (16) is a convex quadratic programming problem of $\mathbf{F}_{i+1}$, which has only one solution for $\mathbf{F}_{i+1}, \gamma_{i+1}=0$ is the only solution to (19b) and (19c) in this case. On other hand, if $\gamma_{i+1}=0$ does not make (19c) satisfied, we have to solve $\operatorname{Tr}\left(\mathbf{F}_{i+1} \mathbf{R}_{\mathbf{x}} \mathbf{F}_{i+1}^{\mathrm{H}}\right)=P_{r}$. It is proved in Appendix I that when $\mathbf{G}_{i+1}$ is fixed, the function

$$
q\left(\gamma_{i+1}\right) \triangleq \operatorname{Tr}\left(\mathbf{F}_{i+1} \mathbf{R}_{\mathbf{x}} \mathbf{F}_{i+1}^{\mathrm{H}}\right)
$$

is a monotonically decreasing function of $\gamma_{i+1}$. Furthermore, it has also been proved that

$$
0 \leq \gamma_{i+1} \leq \sqrt{\frac{\operatorname{Tr}\left(\overline{\mathbf{H}}_{r d}^{\mathrm{H}} \mathbf{G}_{i+1}^{\mathrm{H}} \mathbf{R}_{s} \overline{\mathbf{H}}_{s r}^{\mathrm{H}} \mathbf{R}_{\mathbf{x}}^{-1} \overline{\mathbf{H}}_{s r} \mathbf{R}_{s} \mathbf{G}_{i+1} \overline{\mathbf{H}}_{r d}\right)}{P_{r}}} .
$$

Based on these results, $\gamma_{i+1}$ can be efficiently computed by a one-dimension search, such as bisection search or golden search [11]. Since $\operatorname{Tr}\left(\mathbf{F}_{i+1} \mathbf{R}_{\mathbf{x}} \mathbf{F}_{i+1}^{\mathrm{H}}\right)=P_{r}$ is a stronger condition than $\operatorname{Tr}\left(\mathbf{F}_{i+1} \mathbf{R}_{\mathbf{x}} \mathbf{F}_{i+1}^{\mathrm{H}}\right) \leq P_{r}$, (19c) is satisfied automatically in this case. In summary, the proposed procedure for calculating $\gamma_{i+1}$ is given as follows:

$$
\gamma_{i+1}=\left\{\begin{array}{ll}
0 & \text { if } q(0) \leq P_{r} \\
\text { Solve } q\left(\gamma_{i+1}\right)=P_{r} & \text { Otherwise } \\
\text { using bisection algorithm }
\end{array} .\right.
$$

\section{Summary and convergence analysis}

The proposed iterative algorithm proceeds between (18) and (19a), which can be summarized as Algorithm 1. This iterative algorithm can be shown to converge as follows. It is obvious 
that when $\mathbf{F}_{i}$ is given, the objective function in problem (16) is a convex quadratic function of $\mathbf{G}_{i+1}$. The solution given by (18) corresponds to the minimum MSE for the fixed $\mathbf{F}_{i}$. In other words, $\operatorname{MSE}\left(\mathbf{F}_{i}, \mathbf{G}_{i+1}\right) \leq \operatorname{MSE}\left(\mathbf{F}_{i}, \mathbf{G}_{i}\right)$. On the other hand, when $\mathbf{G}_{i+1}$ is obtained, the optimization problem (16) is a convex quadratic programming problem of $\mathbf{F}_{i+1}$, and the KKT conditions are the necessary and sufficient condition for the optimal solution [9], so we have $\operatorname{MSE}\left(\mathbf{F}_{i+1}, \mathbf{G}_{i+1}\right) \leq$ $\operatorname{MSE}\left(\mathbf{F}_{i}, \mathbf{G}_{i+1}\right)$. It follows that each update on $\mathbf{F}$ or $\mathbf{G}$ will decrease the objective function and thus the iterative algorithm converges.

\section{A Low Complexity Heuristic Approximation for $\gamma_{i}$}

Another heuristic method to compute $\gamma_{i}$ in each iteration is given as follows. It can be shown (due to space limitaion, the proof is not presented here) that for any solution of $\mathbf{F}$ and G satisfying the KKT conditions of the optimization problem (16), the Lagrangian multiplier corresponding to $\mathbf{F}$ and $\mathbf{G}$, satisfies

$$
\lambda=\frac{\operatorname{Tr}\left(\mathbf{G R}_{n_{2}} \mathbf{G}^{\mathrm{H}}\right)}{P_{r}} .
$$

Inspired by [8], in each iteration, to reduce complexity, we use an approximation

$$
\tilde{\gamma}_{i}=\frac{\operatorname{Tr}\left(\mathbf{G}_{i} \mathbf{R}_{n_{2}} \mathbf{G}_{i}^{\mathrm{H}}\right)}{P_{r}}
$$

to replace $\gamma_{i}$ from bisection search. Strictly speaking, (23) only holds when $\mathbf{F}$ and $\mathbf{G}$ satisfy KKT conditions simultaneously. However, in the iterative algorithm, when $\mathbf{F}_{i}$ and $\mathbf{G}_{i}$ have not converged, $\tilde{\gamma}_{i}$ given by (24) may not satisfy (19b). To remedy this problem, [8] proposed to scale $\mathbf{F}_{i}$ by a factor such that $\operatorname{Tr}\left(\mathbf{F}_{i} \mathbf{R}_{\mathbf{x}} \mathbf{F}_{i}^{\mathrm{H}}\right)=P_{r}$ holds. It should also be pointed out that the problem (16) is more general than those in [8]. When the first hop channel is an identity matrix and in the absence of noise, (16) reduces to the problem in [8] and the proposed algorithm also reduces to that in [8].

\section{Simulation Results and Discussions}

In this section, we will investigate the performance of the propose iterative algorithm. In the following, we consider an AF MIMO relay system where the source, relay and destination are equipped with same number of antennas, i.e., $N_{S}=M_{R}=N_{R}=M_{D}=4$. The widely used exponential model [8] is chosen for the channel estimation error covariance matrices:

$$
\begin{aligned}
& \boldsymbol{\Psi}_{s r}=\boldsymbol{\Psi}_{r d}=\left[\begin{array}{cccc}
1 & \alpha & \alpha^{2} & \alpha^{3} \\
\alpha & 1 & \alpha & \alpha^{2} \\
\alpha^{2} & \alpha & 1 & \alpha \\
\alpha^{3} & \alpha^{2} & \alpha & 1
\end{array}\right], \\
& \boldsymbol{\Sigma}_{s r}=\boldsymbol{\Sigma}_{r d}=\sigma_{e}^{2}\left[\begin{array}{cccc}
1 & \beta & \beta^{2} & \beta^{3} \\
\beta & 1 & \beta & \beta^{2} \\
\beta^{2} & \beta & 1 & \beta \\
\beta^{3} & \beta^{2} & \beta & 1
\end{array}\right],
\end{aligned}
$$

where $\alpha$ and $\beta$ are the correlation coefficients, and $\sigma_{e}^{2}$ denotes the estimation error variance.

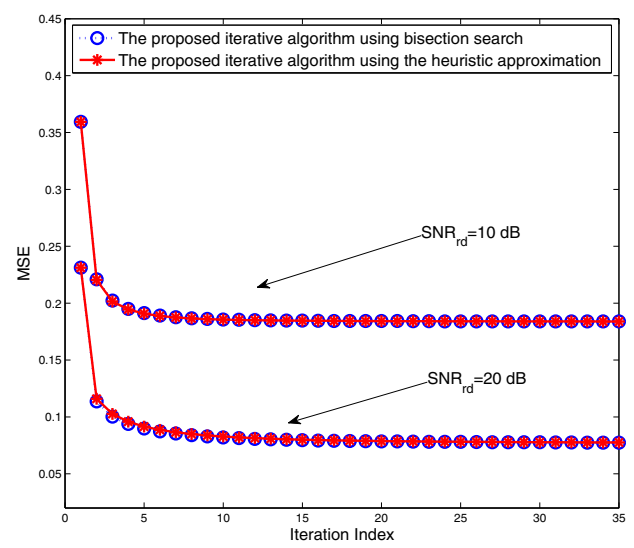

Fig. 2. Convergence performance of the proposed iterative algorithm with $\alpha=0.4, \beta=0.5$ and $\sigma_{e}^{2}=0.002$.

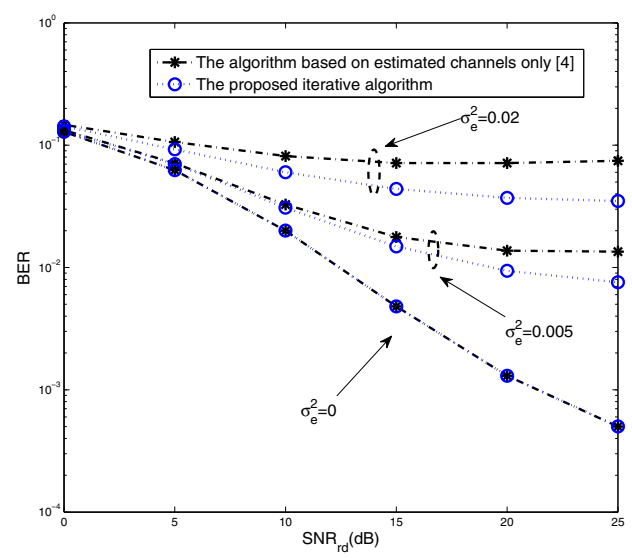

Fig. 3. The BERs for the proposed iterative algorithm and the algorithm based on estimated channels only for different $\sigma_{e}^{2}$, when $\alpha=0.4$ and $\beta=0.5$.

We define the signal-to-noise ratio for the source-relay link $\left(\mathrm{SNR}_{s r}\right)$ as $\mathrm{E}_{s} / \mathrm{N}_{1}=\operatorname{Tr}\left(\mathbf{R}_{s}\right) / \operatorname{Tr}\left(\mathbf{R}_{n_{1}}\right)$, and is fixed as $\mathrm{E}_{s} / \mathrm{N}_{1}=30 \mathrm{~dB}$. At the source, four independent data streams are transmitted by four antennas at the same power. For each data stream, 1000 independent QPSK symbols are transmitted and $\operatorname{Tr}\left(\mathbf{R}_{s}\right)$ is normalized to 1 . The signal-tonoise ratio for the relay-destination link $\left(\mathrm{SNR}_{r d}\right)$ is defined as $\mathrm{E}_{r} / \mathrm{N}_{2}=P_{r} / \operatorname{Tr}\left(\mathbf{R}_{n_{2}}\right)$. The estimated channels, $\overline{\mathbf{H}}_{s r}$ and $\overline{\mathbf{H}}_{r d}$, are generated based on the following distributions

$$
\begin{aligned}
\overline{\mathbf{H}}_{s r} \sim \mathcal{C N}_{M_{R}, N_{S}}\left(\mathbf{0}_{M_{R}, N_{S}}, \frac{\left(1-\sigma_{e}^{2}\right)}{\sigma_{e}^{2}} \boldsymbol{\Sigma}_{s r} \otimes \mathbf{\Psi}_{s r}^{\mathrm{T}}\right), \\
\overline{\mathbf{H}}_{r d} \sim \mathcal{C N}_{M_{D}, N_{R}}\left(\mathbf{0}_{M_{D}, N_{R}}, \frac{\left(1-\sigma_{e}^{2}\right)}{\sigma_{e}^{2}} \boldsymbol{\Sigma}_{r d} \otimes \mathbf{\Psi}_{r d}^{\mathrm{T}}\right),
\end{aligned}
$$

such that channel realizations $\mathbf{H}_{s r}=\overline{\mathbf{H}}_{s r}+\Delta \mathbf{H}_{s r}$ and $\mathbf{H}_{r d}=$ $\overline{\mathbf{H}}_{r d}+\Delta \mathbf{H}_{r d}$ have unit variance. In the following figures, MSE is referred to as the simulated MSE of the equalized signal at the destination. Each point in the following figures is an average of 10000 independent channel realizations. 
Fig. 2 shows the MSE performances of the proposed iterative algorithm as a function of iteration index with $\mathrm{SNR}_{r d}=$ $10 \mathrm{~dB}$ and $\mathrm{SNR}_{r d}=20 \mathrm{~dB}$. The correlation coefficients are set as $\alpha=0.4, \beta=0.5$ and $\sigma_{e}^{2}=0.002$. The threshold, $\epsilon$, for terminating the iterative algorithm is $0.0001 \operatorname{Tr}\left(\mathbf{F}_{i} \mathbf{F}_{i}^{\mathrm{H}}\right)$. From the figure, it can be seen that the iterative algorithm converges to a stable solution after around 15 iterations regardless of $\mathrm{SNR}_{r d}$. It can also be seen that the heuristic approximation, although derived in an ad-hoc fashion, provides a similar performance to the bisection search method.

Fig. 3 compares the bit error rates (BERs) of the proposed iterative algorithm and the algorithm based on estimated channel only [4] as a function of $\mathrm{SNR}_{r d}$, when $\alpha=0.4$ and $\beta=0.5$. It can be seen that the BER performances improve when $\sigma_{e}^{2}$ decreases. It can also be seen that for different $\sigma_{e}^{2}$, the performance of the proposed iterative algorithm is always better than that of the algorithm based on estimated channels only.

\section{CONCLUSIONS}

In this paper, the LMMSE transceiver design for dualhop AF MIMO relay systems with Gaussian random channel uncertainties in both hops was considered. The data MSE expression at the destination averaged over the random channel uncertainties was first derived. Then the robust iterative algorithm was proposed to minimize the average MSE. Simulation results showed that the proposed iterative algorithm performs better than the existing algorithm without taking the channel uncertainties into account.

\section{APPENDIX I}

PROOF OF $q\left(\gamma_{i+1}\right)$ IS MONOTONICALLY DECREASING AND

$$
\text { UPPER BOUND ON } \gamma_{i+1}
$$

First, we note the following two well-known results for matrix.

Property 1: For any two positive Hermitian matrices, $\mathbf{M}$ and $\overline{\mathbf{N}, \mathbf{M} \succeq \mathbf{N}}$ if and only if $\mathbf{M}^{-1} \preceq \mathbf{N}^{-1}[12,7.7 .4]$.

Property 2: For any two positive semi-definite Hermitian $\overline{\text { matrices, } \mathbf{M}}$ and $\mathbf{N}, \operatorname{Tr}(\mathbf{M N}) \geq 0$.

For notational simplicity, define

$$
\begin{aligned}
\mathbf{A}_{i+1} & =\overline{\mathbf{H}}_{r d}^{\mathrm{H}} \mathbf{G}_{i+1}^{\mathrm{H}} \mathbf{G}_{i+1} \overline{\mathbf{H}}_{r d}+\operatorname{Tr}\left(\mathbf{G}_{i+1} \boldsymbol{\Sigma}_{r d} \mathbf{G}_{i+1}^{\mathrm{H}}\right) \mathbf{\Psi}_{r d} \\
\mathbf{B}_{i+1} & =\overline{\mathbf{H}}_{r d}^{\mathrm{H}} \mathbf{G}_{i+1}^{\mathrm{H}} \mathbf{R}_{s} \overline{\mathbf{H}}_{s r}^{\mathrm{H}} \mathbf{R}_{\mathbf{x}}^{-1} \overline{\mathbf{H}}_{s r} \mathbf{R}_{s} \mathbf{G}_{i+1} \overline{\mathbf{H}}_{r d}
\end{aligned}
$$

Notice that $\mathbf{A}_{i+1}$ is a positive definite Hermitian matrix and $\mathbf{B}_{i+1}$ is a positive semi-definite Hermitian matrix. Putting (26) and (27) into the definition of $q\left(\gamma_{i+1}\right)$ in (20), we have

$$
q\left(\gamma_{i+1}\right) \triangleq \operatorname{Tr}\left(\mathbf{F}_{i+1} \mathbf{R}_{\mathbf{x}} \mathbf{F}_{i+1}^{\mathrm{H}}\right)=\operatorname{Tr}\left(\left(\mathbf{A}_{i+1}+\gamma_{i+1} \mathbf{I}\right)^{-2} \mathbf{B}_{i+1}\right) .
$$

For any two values $a$ and $b$, with $a \geq b \geq 0$,

$$
q(b)-q(a)=\operatorname{Tr}\left(\left(\left(\mathbf{A}_{i+1}+b \mathbf{I}\right)^{-2}-\left(\mathbf{A}_{i+1}+a \mathbf{I}\right)^{-2}\right) \mathbf{B}_{i+1}\right) .
$$

Below we will show that $q(b)-q(a) \geq 0$. Because $\mathbf{A}_{i+1}$ is a positive semi-definite Hermitian matrix and $a \geq b,\left(\mathbf{A}_{i+1}+\right.$ $b \mathbf{I})^{2} \preceq\left(\mathbf{A}_{i+1}+a \mathbf{I}\right)^{2}$, and with property 1, we have

$$
\left(\mathbf{A}_{i+1}+b \mathbf{I}\right)^{-2}-\left(\mathbf{A}_{i+1}+a \mathbf{I}\right)^{-2} \succeq 0 .
$$

Together with the fact that $\mathbf{B}_{i+1}$ is a positive semi-definite Hermitian matrix, based on property 2 , we have

$$
\operatorname{Tr}\left(\left(\left(\mathbf{A}_{i+1}+b \mathbf{I}\right)^{-2}-\left(\mathbf{A}_{i+1}+a \mathbf{I}\right)^{-2}\right) \mathbf{B}_{i+1}\right) \geq 0,
$$

therefore, $q(b)-q(a) \geq 0$, and $q\left(\gamma_{i+1}\right)$ is a monotonically decreasing function of $\gamma_{i+1}$.

With regard to the upper bound of $\gamma_{i+1}$, it is obvious that

$$
\left(\mathbf{A}_{i+1}+\gamma_{i+1} \mathbf{I}\right)^{2} \succ \gamma_{i+1}^{2} \mathbf{I} \text {. }
$$

As mentioned in the proposed procedure (22) for computing $\gamma_{i+1}$, the bisection algorithm is only needed when $\gamma_{i+1} \neq 0$. So we can invert both side of (32), multiple $\mathbf{B}_{i+1}$ from the right and take the trace of the both sides, and it follows that

$$
\operatorname{Tr}\left(\mathbf{F}_{i+1} \mathbf{R}_{\mathbf{x}} \mathbf{F}_{i+1}^{\mathrm{H}}\right)<\frac{1}{\gamma_{i+1}^{2}} \operatorname{Tr}\left(\mathbf{B}_{i+1}\right) .
$$

Notice that based on (19b), if $\gamma_{i+1} \neq 0, \operatorname{Tr}\left(\mathbf{F}_{i+1} \mathbf{R}_{\mathbf{x}} \mathbf{F}_{i+1}^{\mathrm{H}}\right)=$ $P_{r}$ and then

$$
\gamma_{i+1}<\sqrt{\frac{\operatorname{Tr}\left(\mathbf{B}_{i+1}\right)}{P_{r}}},
$$

which is exactly the expression given in (21).

\section{ACKNOWLEDGEMENT}

This study was partially supported by a grant from the Research Grants Council of the Hong Kong SAR.

\section{REFERENCES}

[1] J. N. Laneman, D. N. C. Tse, and G. W. Wornell, "Cooperative diversity in wireless networks: Efficient protocols and outage behavior,' IEEE Trans. Information Theory, vol. 50, no. 12, pp. 3062-3080, Dec. 2004.

[2] E. G. Larsson and P. Stoica, Space-Time Block Coding for Wireless Communications. Cambridge University Press, 2003.

[3] O. Munoz-Medina, J. Vidal, and A. Agustin, "Linear transceiver design in nonregenerative relays with channel state information," IEEE Trans. Signal Processing, vol. 55, no. 6, pp. 2198-2206, June, 2007.

[4] W. Guan and H. W. Luo, "Joint MMSE transceiver design in nonregenerative MIMO relay systems," IEEE Communications Letters, vol. 12, no. 7, pp. 517-519, July, 2008.

[5] A. S. Behbahani, R. Merched, and A. M. Eltawil, "Optimizations of a MIMO relay Network, "IEEE Trans. Signal Processing, vol. 56, no. 10, part 2, pp. 5062-5073, Oct. 2008.

[6] A. K. Gupta and D. K. Nagar, Matrix Variates Distributions. London, U.K., Chapman\&Hall/CRC, 2000.

[7] A. T. James, "Dstributions of matrix variates and latent roots derived from normal samples," Ann. Math. Statistics, vol. 35, pp. 475-501, 1964.

[8] M. Ding and S. D. Blostein, "MIMO minimum total MSE transceiver design with imperfect CSI at both ends," IEEE Trans. Signal Processing, vol. 57, no. 3, pp. 1141-1150, March 2009.

[9] D. P. Bertsekas, Nonlinear Programming, 2nd ed. New York: Athena, 2005.

[10] J. Zhang, Y. Wu, S. Zhou, and J. Wang, "Joint linear transmitter and receiver design for the downlink of multiuser MIMO systems," IEEE Communications Letters, vol. 9, no. 11, pp. 991-993, Nov, 2005.

[11] E. K. P. Chong and S. H. Zak, An Introduction to Optimization. John wiley \& Sons, Inc. 1996.

[12] R. A. Horn and C. R. Johnson, Matrix Analysis. Cambridge University Press, 1985. 